\title{
Determination of molecular dipole orientation in doped fluorescent organic thin films by photoluminescence measurements
}

Cite as: Appl. Phys. Lett. 96, 073302 (2010); https://doi.org/10.1063/1.3309705

Submitted: 11 December 2009 . Accepted: 16 January 2010 . Published Online: 17 February 2010

Jörg Frischeisen, Daisuke Yokoyama, Chihaya Adachi, and Wolfgang Brütting

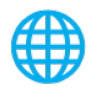

\section{ARTICLES YOU MAY BE INTERESTED IN}

Light extraction and optical loss mechanisms in organic light-emitting diodes: Influence of the emitter quantum efficiency

Journal of Applied Physics 104, 123109 (2008); https://doi.org/10.1063/1.3043800

Nearly $100 \%$ internal phosphorescence efficiency in an organic light-emitting device Journal of Applied Physics 90, 5048 (2001); https://doi.org/10.1063/1.1409582

Evidence for non-isotropic emitter orientation in a red phosphorescent organic lightemitting diode and its implications for determining the emitter's radiative quantum efficiency

Applied Physics Letters 99, 163302 (2011); https://doi.org/10.1063/1.3653475

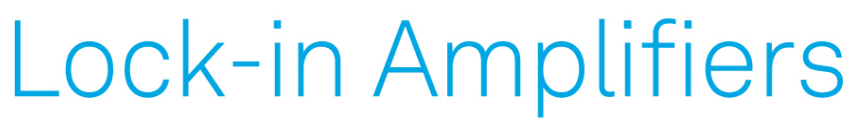

... and more, from DC to $600 \mathrm{MHz}$ Watch 


\title{
Determination of molecular dipole orientation in doped fluorescent organic thin films by photoluminescence measurements
}

\author{
Jörg Frischeisen, ${ }^{1, a)}$ Daisuke Yokoyama, ${ }^{2, b)}$ Chihaya Adachi, ${ }^{2}$ and Wolfgang Brütting ${ }^{1, c)}$ \\ ${ }^{1}$ Institute of Physics, University of Augsburg, Universitätsstraße 1, 86159 Augsburg, Germany \\ ${ }^{2}$ Center for Future Chemistry, Kyushu University, 744 Motooka, Nishi, Fukuoka 819-0395, Japan
}

(Received 11 December 2009; accepted 16 January 2010; published online 17 February 2010)

\begin{abstract}
The orientation of the transition dipole moments of fluorescent organic molecules doped into a matrix material is determined by photoluminescence measurements of the angular dependent emission spectra and by comparison with simulations. The analysis of two small molecular materials doped into a 4,4'-bis(N-carbazole)-biphenyl matrix is demonstrated, yielding a horizontal orientation of $91 \%$ for 4,4'-bis[4-(diphenylamino)styryl]biphenyl and a completely random orientation in case of tris-(8-hydroxyquinoline)aluminum $\left(\mathrm{Alq}_{3}\right)$. This expeditious technique does not require detailed information about the optical properties of the dopant, making this method particularly suitable for characterizing newly developed materials for organic light-emitting diodes with enhanced light-outcoupling efficiency. (C) 2010 American Institute of Physics.
\end{abstract}

[doi:10.1063/1.3309705]

Organic light-emitting diodes (OLEDs) have been the subject of intensive studies and continually improved due to their large potential for power efficient lighting applications. In particular, OLEDs based on small molecular emitters doped into a matrix material show exceptionally high efficiencies, already reaching fluorescent tube efficiency. ${ }^{1}$ Huge potential for further improvement is achievable by the employment of molecular materials with a horizontal orientation of the transition dipole moments, i.e., emissive dipoles lying parallel to the substrate. Numerical simulations show that depending on the OLED structure, the outcoupling efficiency can be increased by a factor of 1.5 compared to a random dipole orientation. ${ }^{2,3}$ In contrast to polymer OLEDs, it is generally believed that the emitting materials utilized in small molecule based OLEDs usually show isotropic orientation. There are, however, some reports about horizontal orientation in neat films of amorphous low-molecular weight materials ${ }^{4}$ and the general relationship between molecular structure and orientation has been pointed out. ${ }^{5,6}$ Furthermore, the horizontal orientation of fluorescent molecules in doped films has already been demonstrated, ${ }^{6}$ opening up new possibilities for device improvement. In addition, the vertical charge transport in devices using flat-lying molecules can be enhanced. $^{7}$

Considering the enormous potential of horizontally oriented emitters in doped films, it is essential to develop reliable and straightforward methods in order to unambiguously determine the dipole orientation of emitter materials. In principle, ellipsometric measurements yield information about the molecular orientation and its transition dipole moment, however, ellipsometry is usually not sensitive enough to detect small amounts of dopants within a matrix. Several additional techniques have been proposed: Edge emission measurements, ${ }^{4,6}$ surface-plasmon-coupled emission, ${ }^{8}$ and investigation of dye molecules in microcavity structures. ${ }^{9}$

\footnotetext{
${ }^{a)}$ Electronic mail: joerg.frischeisen@physik.uni-augsburg.de.

${ }^{b}$ Present address: Graduate School of Science and Engineering, Yamagata University, 4-13-16 Johnan, Yonezawa, Yamagata 992-8510, Japan.

${ }^{c}$ Electronic mail: wolfgang.bruetting@ @ physik.uni-augsburg.de.
}

Though these methods work in principle, the extraction of the relevant information on molecular orientation is not straightforward.

In this study, we present a procedure that is based on the angular dependent measurement of the photoluminescence (PL) emission spectrum of a specific layer structure and the comparison to numerical simulations. With this technique also the degree of horizontal orientation can be determined in the case of only partially oriented dipoles. The analysis is applied to the investigation of the blue emitter 4,4'-bis[4-(diphenylamino)styryl]biphenyl (BDASBi) in a 4,4'-bis(N-carbazole)-biphenyl (CBP) matrix. The chemical structure of BDASBi is shown in Fig. 1(a). From a chemical point of view, BDASBi is quite similar to $4,4^{\prime}$ bis[(N-carbazole)styryl]biphenyl (BSB-Cz), which is known to exhibit mainly horizontally oriented dipoles in neat and doped films. ${ }^{6}$ Thus, it is expected that the emitting dipoles in BDASBi are oriented predominantly parallel to the substrate plane as well.

Figures 1(b) and 1(c) illustrate the coordinate system and the definition of emission angle used in the following analysis. Materials with random transition dipole orientation can be treated as a superposition of $p_{\mathrm{x}^{-}}, p_{\mathrm{y}^{-}}$and $p_{\mathrm{z}}$-dipoles (one (a)

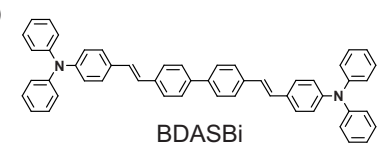

(c)

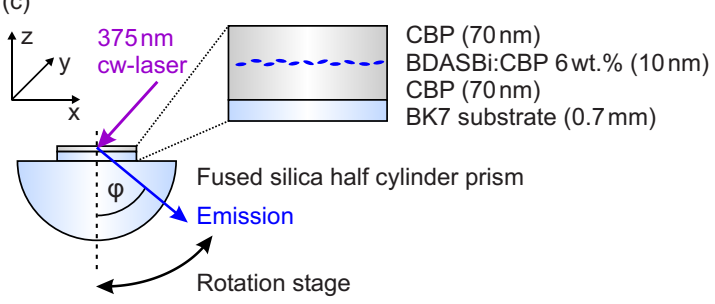

(b)

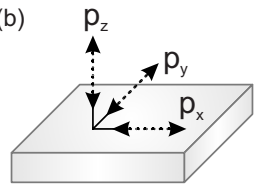

$\%(10 \mathrm{~nm})$
FIG. 1. (Color online) (a) Chemical structure of BDASBi. (b) Definition of dipole orientations. (c) Experimental setup and cross-sectional schematic view of the layer structure. 

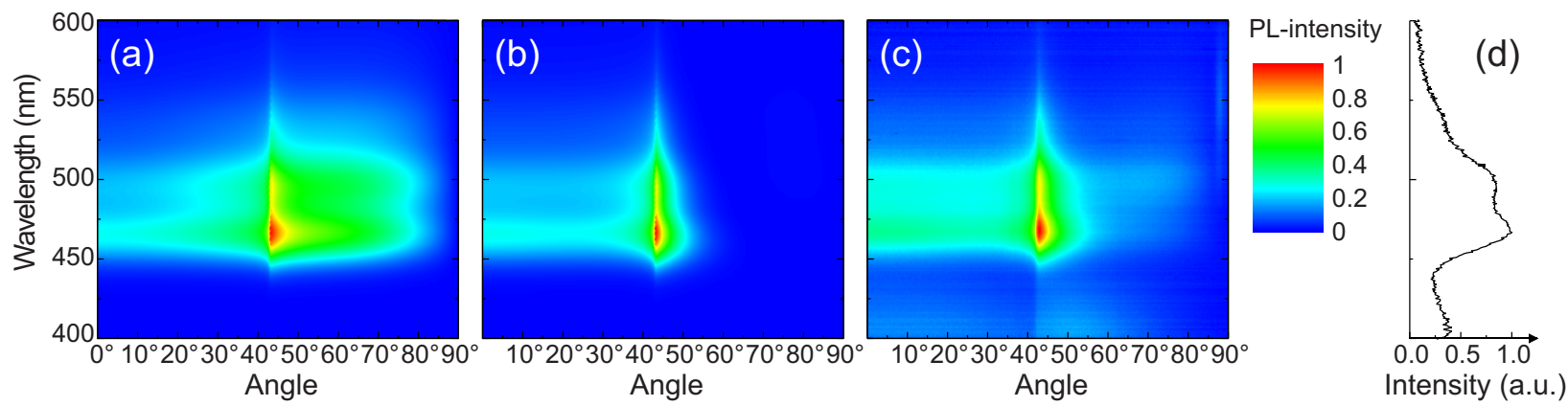

FIG. 2. (Color online) (a) Simulated p-polarized angular dependent emission spectrum coupled out into the half cylinder prism for a random dipole orientation. (b) Analogous simulation for horizontally oriented dipoles. (c) Measured p-polarized emission spectrum of the device shown in Fig. 1(c). (d) Spectrum of BDASBi doped into CBP extracted from Fig. 2(c).

third each), while materials with horizontally oriented dipoles are considered as consisting of one half of $p_{\mathrm{x}}$ - and one half of $p_{\mathrm{y}}$-dipoles. Without loss of generality, the emission into the X-z-plane is considered. Hence, the $p_{\mathrm{y}}$-dipoles emit only s-polarized light whereas the $p_{\mathrm{x}^{-}}$and $p_{\mathrm{z}}$-dipoles are responsible for the $p$-polarized emission. Consequently, the analysis of the $p$-polarized emission can yield information about the existence of vertical dipoles. Taking into account that dipoles radiate strongest perpendicular to their direction of oscillation, the $p_{\mathrm{z}}$-dipoles emit mainly at large angles. In an OLED structure, this corresponds to strong coupling to waveguide modes inside the organic layers or to the excitation of surface plasmon polaritons at the organic-metal-interface. ${ }^{10,11}$ This is the reason why the outcoupling efficiency can be increased as soon as there are only horizontally oriented dipoles and no vertical $p_{\mathrm{z}}$-dipoles.

The stack investigated in this study is shown in Fig. 1(c). A $10 \mathrm{~nm}$ thick BDASBi:CBP (6 wt \%) layer is positioned in between two $70 \mathrm{~nm}$ thick CBP layers. All layers were thermally evaporated in a vacuum chamber and encapsulated under inert gas atmosphere. A $375 \mathrm{~nm} \mathrm{cw}$-laser diode incident under $45^{\circ}$ was used as excitation source. Since CBP has only a small absorption at this wavelength, emission from the matrix is suppressed. The angular dependent PL spectrum from $0^{\circ}$ to $90^{\circ}$ was measured with a fiber optical spectrometer and a polarizer to distinguish between $s$ - and $p$-polarized emissions. The thickness of this layer structure was chosen based on optical simulations (details see below) so that the difference in the emission spectrum is clearly pronounced depending on whether the orientation of the emitting dipoles is random or exclusively in the substrate plane. The structure has to be free of metallic layers, because otherwise the $p_{7}$-dipoles couple almost completely to surface plasmon polaritons. ${ }^{12}$ In this case, no influence on the shape of the PL spectrum could be detected. Furthermore, the doped region should be narrow, as the simulation assumes a discrete emitter position.

The simulation is based on the dipole model developed by Chance, Prock, and Silbey ${ }^{13}$ and later on extended by Barnes. ${ }^{14}$ A detailed explanation can be found in Ref. 15. All three dipole orientations were simulated separately, permitting subsequent weighting for arbitrary dipole distributions. The only requirements for the simulation are the optical constants of the CBP matrix, the PL spectrum of the doped layer and the total thickness, which was checked by ellipsometry. The small birefringence of the CBP matrix ${ }^{6}$ is not taken into account, as this considerably increases the complexity of the simulation without changing the overall result significantly. It is noteworthy that the optical constants of the dopant are not required, emphasizing that this technique is exceptionally useful for the analysis of newly developed materials with unknown optical properties.

The simulated $p$-polarized spectra are shown in Figs. 2(a) and 2(b) for randomly and horizontally oriented dipoles, respectively. The influence of $p_{z}$-dipoles on the emission at large angles is clearly observable. A comparison with the measured spectrum presented in Fig. 2(c) indicates that the BDASBi transition dipole moments are primarily horizontally oriented. In addition, the spectrum of BDASBi extracted from the measurement is displayed in Fig. 2(d).

In order to determine the exact fraction of horizontally oriented dipoles, a cross section at $480 \mathrm{~nm}$ was investigated, cf. Fig. 3(a). The cross sections are normalized to the emission at small angles because the intensity in this range is not affected by the amount of vertical dipoles. It should be noted that not even the PL spectrum of the dopant is necessary for this analysis because the simulated cross sections depend
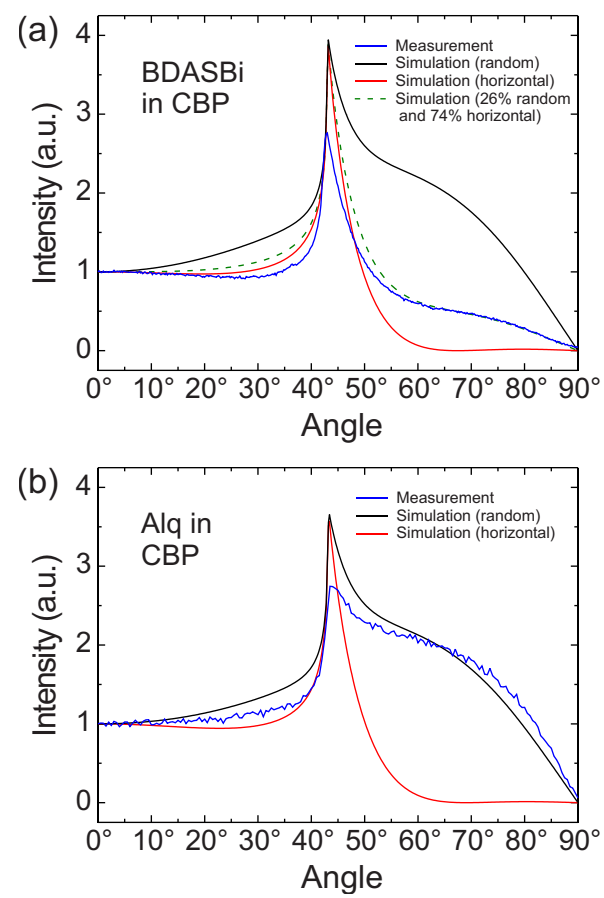

FIG. 3. (Color online) (a) Cross sections at $480 \mathrm{~nm}$ of the spectra shown in Fig. 2 normalized to the emission at small angles. The fit was obtained by weighting the simulations for random and horizontal dipole orientation by 0.26 and 0.74 , respectively. (b) Cross section at $520 \mathrm{~nm}$ of a measurement with $\mathrm{Alq}_{3}$ doped into a comparable layer structure as shown in Fig. 1(c) with around $170 \mathrm{~nm}$ total CBP thickness. 
solely on the optical constants of the matrix material as long as the dopant ratio is small. The measured curve lies between the two simulated curves for random and entirely horizontal dipole orientation. The deviation at angles close to the peak is probably caused by the finite angular resolution of the experimental setup. A good agreement between measurement and simulation for large angles at which the emission from the vertical dipoles is most prominent can be obtained by weighting the simulations for randomly and horizontally oriented dipoles with 0.26 and 0.74 , respectively. This amounts to a total fraction of $91 \%$ horizontally oriented $p_{\mathrm{x}^{-}}$and $p_{\mathrm{y}}$-dipoles and $9 \%$ vertical $p_{\mathrm{z}}$-dipoles with an error of about $\pm 4 \%$. Hence, the BDASBi transition dipole moments in a CBP matrix are predominantly oriented horizontally.

To verify that the proposed analysis is suitable for the determination of the dipole orientation the cross section at $520 \mathrm{~nm}$ for a reference sample with tris-(8hydroxyquinoline)aluminum $\left(\mathrm{Alq}_{3}\right)$ doped into a CBP matrix is shown in Fig. 3(b). In this case, the measured curve can be well described by the simulation for completely random dipole orientation as expected for a material which is known to have isotropic orientation of the molecules. ${ }^{4}$

In summary, a method to determine the orientation of the transition dipole moments of a dopant in organic thin films was demonstrated. This technique is based on the measurement of the angular dependent PL spectrum and the comparison with simulations. In contrast to other procedures, this is a straightforward and expeditious approach that does not only result in a rough estimate but quantitatively determines the fraction of horizontally oriented dipoles in doped films. We want to emphasize that it is not necessary to know any information about the dopant for the detailed analysis of the cross sections. Neither the optical constants nor the PLspectrum are required, rendering this method particularly useful for a reliable investigation of newly developed materials. The convenience of this technique was demonstrated by the investigation of BDASBi doped into a CBP matrix, resulting in a horizontal dipole orientation of $91 \%$. It should be noted that this approach is in principle also applicable to neat films as well as phosphorescent emitters. Considering the decisive influence of the dipole orientation on the outcoupling efficiency of OLEDs, this is an important contribution to the characterization of emitter materials and the further enhancement of organic light sources.

We acknowledge financial support by the Elite Network of Bavaria through the international graduate school "Materials Science of Complex Interfaces" and by the Japan Society for the Promotion of Science (JSPS).

${ }^{1}$ S. Reineke, F. Lindner, G. Schwartz, N. Seidler, K. Walzer, B. Lüssem, and K. Leo, Nature (London) 459, 234 (2009).

${ }^{2}$ J.-S. Kim, P. K. H. Ho, N. C. Greenham, and R. H. Friend, J. Appl. Phys. 88, 1073 (2000).

${ }^{3}$ S. Nowy, J. Frischeisen, and W. Brütting, Proc. SPIE 7415, 74151C (2009).

${ }^{4}$ H.-W. Lin, C.-L. Lin, H.-H. Chang, Y.-T. Lin, C.-C. Wu, Y.-M. Chen, R.-T. Chen, Y.-Y. Chien, and K.-T. Wong, J. Appl. Phys. 95, 881 (2004).

${ }^{5}$ D. Yokoyama, A. Sakaguchi, M. Suzuki, and C. Adachi, Appl. Phys. Lett. 93, 173302 (2008).

${ }^{6}$ D. Yokoyama, A. Sakaguchi, M. Suzuki, and C. Adachi, Org. Electron. 10, 127 (2009).

${ }^{7}$ D. Yokoyama, Y. Setoguchi, A. Sakaguchi, M. Suzuki, and C. Adachi, Adv. Funct. Mater. 20, 386 (2010).

${ }^{8}$ H. M. Hiep, M. Fujii, and S. Hayashi, Appl. Phys. Lett. 91, 183110 (2007).

${ }^{9}$ S. H. Garrett, J. A. E. Wasey, and W. L. Barnes, J. Mod. Opt. 51, 2287 (2004).

${ }^{10}$ L. H. Smith, J. A. E. Wasey, I. D. W. Samuel, and W. L. Barnes, Adv. Funct. Mater. 15, 1839 (2005).

${ }^{11}$ J. M. Ziebarth and M. D. McGehee, J. Appl. Phys. 97, 064502 (2005).

${ }^{12}$ W. H. Weber and C. F. Eagen, Opt. Lett. 4, 236 (1979).

${ }^{13}$ R. R. Chance, A. Prock, and R. Silbey, J. Chem. Phys. 60, 2744 (1974).

${ }^{14}$ W. L. Barnes, J. Mod. Opt. 45, 661 (1998).

${ }^{15}$ S. Nowy, B. C. Krummacher, J. Frischeisen, N. A. Reinke, and W. Brütting, J. Appl. Phys. 104, 123109 (2008). 